\title{
Comparison of in-brace correction between a (TLSO) using a floating pad with adjustable straps and a standard anterior open TLSO with no window openings for the treatment of congenital and infantile scoliosis: a case series
}

\author{
David Speers", Katie Fisher
}

From 10th International Conference on Conservative Management of Spinal Deformities - SOSORT 2013

Annual Meeting

Chicago, IL, USA. 8-11 May 2013

\section{Background}

Many styles of TLSOs can be used in the treatment of congenital and infantile scoliosis. A standard anterior open TLSO design with no window openings is one of the more common styles. A specific TLSO called the "Kalibus" was developed for the treatment of congenital scoliosis in very young children. The Kalibus consists of a rigid pelvic section, a floating pad with adjustable tension straps with a large opening opposite the pad and a rigid shoulder cuff. Typically, an inflexible spine characterizes congenital scoliosis, however, evaluating supine traction $\mathrm{x}$-rays can determine spine flexibility as well as whether bracing is a treatment option to reduce some of the flexible deformities of the curve(s).

\section{Purpose}

This study compared the in-brace $\mathrm{x}$-ray correction between a Kalibus-style TLSO and a standard anterior open design with no window openings.

\section{Methods}

For this study, three children (ages 2 years 8 months, 20 months and 11 months), two with a diagnosis of congenital scoliosis with some flexibility observed in traction $\mathrm{x}$-rays and one with infantile scoliosis, were treated with both a Kalibus-style TLSO and a standard anterior open

* Correspondence: david.speers@scheckandsiress.com Scheck and Siress Orthotics and Prosthetics, Chicago, IL, USA
TLSO with no window openings. Initial in-brace $\mathrm{x}$-rays were evaluated by one orthopedic surgeon.

\section{Results}

The standard anterior open TLSO provided corrections of 7 degrees and 8 degrees in the congenital scoliosis patients and 3 degrees in the infantile scoliosis patient. The Kalibus-style TLSO provided corrections of 0 degrees and 7 degrees in the congenital scoliosis patients and 0 degrees in the infantile scoliosis patient.

\section{Conclusions and discussion}

In this case series of three patients, the standard anterior open TLSO with no window openings produced greater in-brace correction than the Kalibus-style TLSO with a floating, adjustable pad with an opening opposite the pad. Additionally, we found that parents preferred the standard anterior open design as it was easier to manage than the Kalibus style.

Published: 18 September 2013

\section{Reference}

1. Trobisch PD, Samdani A, Oâ€TMNeil C, Betz R, Cahill P: Therapeutic success of conservative therapy in severe infantile idiopathic scoliosis. Journal of Ortho and Traumatology 2012, 150(1):52-55.

doi:10.1186/1748-7161-8-S2-O52

Cite this article as: Speers and Fisher: Comparison of in-brace correction between a (TLSO) using a floating pad with adjustable straps and a standard anterior open TLSO with no window openings for the treatment of congenital and infantile scoliosis: a case series. Scoliosis 2013 8(Suppl 2):052. 